\title{
Söll, Matthias
}

\section{Orientierungspotenziale des Basiscurriculums der Berufs- und} Wirtschaftspädagogik für die disziplinäre Binnen- und

\section{Außenlegitimität}

Seifried, Jürgen [Hrsg.]; Seeber, Susan [Hrsg.]; Ziegler, Birgit [Hrsg.]: Jahrbuch der berufs- und wirtschaftspädagogischen Forschung 2017. Opladen ; Berlin ; Toronto : Verlag Barbara Budrich 2017, S. 29-42. (Schriftenreihe der Sektion Berufs- und Wirtschaftspädagogik der Deutschen Gesellschaft für Erziehungswissenschaft (DGfE))

Quellenangabe/ Reference:

Söll, Matthias: Orientierungspotenziale des Basiscurriculums der Berufs- und Wirtschaftspädagogik für die disziplinäre Binnen- und Außenlegitimität - In: Seifried, Jürgen [Hrsg.]; Seeber, Susan [Hrsg.]; Ziegler, Birgit [Hrsg.]: Jahrbuch der berufs- und wirtschaftspädagogischen Forschung 2017. Opladen ; Berlin ; Toronto : Verlag Barbara Budrich 2017, S. 29-42 - URN:

urn:nbn:de:0111-pedocs-184152 - DOI: 10.25656/01:18415

https://nbn-resolving.org/urn:nbn:de:0111-pedocs-184152

https://doi.org/10.25656/01:18415

in Kooperation mit / in cooperation with:

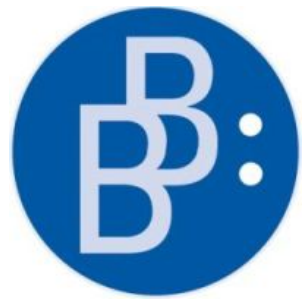

https://www.budrich.de

\section{Nutzungsbedingungen}

Gewährt wird ein nicht exklusives, nicht übertragbares, persönliches und beschränktes Recht auf Nutzung dieses Dokuments. Dieses Dokument ist ausschließlich für den persönlichen, nicht-kommerziellen Gebrauch bestimmt. Die Nutzung stellt keine Übertragung des Eigentumsrechts an diesem Dokument dar und gilt vorbehaltlich der folgenden Einschränkungen: Auf sämtlichen Kopien dieses Dokuments müssen alle Urheberrechtshinweise und sonstigen Hinweise auf gesetzlichen Schutz beibehalten werden. Sie dürfen dieses Dokument nicht in irgendeiner Weise abändern, noch dürfen Sie dieses Dokument für öffentliche oder kommerzielle Zwecke vervielfältigen, öffentlich ausstellen, aufführen, vertreiben oder anderweitig nutzen

Mit der Verwendung dieses Dokuments erkennen Sie die Nutzungsbedingungen an.

\section{Terms of use}

We grant a non-exclusive, non-transferable, individual and limited right to using this document.

This document is solely intended for your personal, non-commercial use. Use of this document does not include any transfer of property rights and it is conditional to the following limitations: All of the copies of this documents must retain all copyright information and other information regarding legal protection. You are not allowed to alter this document in any way, to copy it for public or commercial purposes, to exhibit the document in public, to perform, distribute or otherwise use the document in public.

By using this particular document, you accept the above-stated conditions of use.

\section{Kontakt / Contact:}

\section{peDOCS}

DIPF | Leibniz-Institut für Bildungsforschung und Bildungsinformation

Informationszentrum (IZ) Bildung

E-Mail: pedocs@dipf.de

Internet: www.pedocs.de

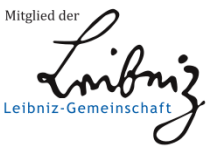


Schriftenreihe der Sektion

Berufs- und Wirtschaftspädagogik

der Deutschen Gesellschaft

für Erziehungswissenschaft (DGfE) 
Jürgen Seifried

Susan Seeber

Birgit Ziegler (Hrsg.)

\section{Jahrbuch der berufs- und} wirtschaftspädagogischen Forschung 2017

Verlag Barbara Budrich

Opladen • Berlin • Toronto 2017 
Bibliografische Information der Deutschen Nationalbibliothek

Die Deutsche Nationalbibliothek verzeichnet diese Publikation in der Deutschen

Nationalbibliografie; detaillierte bibliografische Daten sind im Internet über

http://dnb.d-nb.de abrufbar.

Gedruckt auf säurefreiem und alterungsbeständigem Papier

Alle Rechte vorbehalten

(C2017 Verlag Barbara Budrich, Opladen, Berlin \&Toronto

www.budrich-verlag.de

ISBN 978-3-8474-2141-2 (Paperback)

eISBN 978-3-8474-1131-4 ( eBook)

Das Werk einschließlich aller seiner Teile ist urheberrechtlich geschützt. Jede Verwertung außerhalb der engen Grenzen des Urheberrechtsgesetzes ist ohne Zustimmung des Verlages unzulässig und strafbar. Das gilt insbesondere für Vervielfältigungen, Übersetzungen, Mikroverfilmungen und die Einspeicherung und Verarbeitung in elektronischen Systemen.

Umschlaggestaltung: Bettina Lehfeldt, Kleinmachnow - www.lehfeldtgraphic.de Typographisches Lektorat: Anja Borkam, Jena 


\section{Inhaltsverzeichnis}

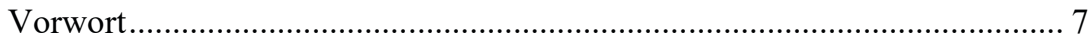

\section{Teil I: Überlegungen zum disziplinären Selbstverständis der Berufs- und Wirtschaftspädagogik}

Karin Büchter

Zum Gehalt berufs- und wirtschaftspädagogischer

Selbstthematisierungen - Rückblick und Ausblick

Matthias Söll

Orientierungspotenziale des Basiscurriculums der Berufs- und

Wirtschaftspädagogik für die disziplinäre Binnen- und Außenlegitimität.... 29

\section{Teil II: Berufliche Lehr-Lern- und Unterrichtsforschung}

Christoph Helm, Jacqueline Netzthaler und Bettina Kreuzer

Kooperatives Lernen im kaufmännischen Unterricht. Eine

Netzwerkanalyse zu sozial-konstruktivistischen Lerntheorien 43

Manuela Niethammer und Anke Langner

Inklusion als fachdidaktischer Anspruch

Svenja Ohlemann und Katja Driesel-Lange

Individuelle Begleitung beruflicher Entwicklung: Kompetenzförderung anhand von Lernstilen.

Mandy Hommel, Bärbel Fürstenau, Claudia Leopold, Héctor Ponce und Mario López

Beitrag von Banken-Webseiten zur Entwicklung der Finanzkompetenz

potentieller Darlehensnehmer/innen über Baufinanzierungen

\section{Teil III: Hochschul- und Lehrerbildungsforschung}

Silke Lange und Dietmar Frommberger

Zur Ausgestaltung schulischer Praxisphasen im beruflichen

Lehramtsstudium - Ergebnisse einer ersten Analyse. 
Heike Jahncke und Karina Kiepe

Handlungsempfehlungen aus dem Einsatz und der Evaluation eines

Tagungsportfolios im Rahmen der Lehrerbildung

Julia Warwas und Andreas Rausch

Unterrichtliche Überzeugungen und Praktiken von Lehrkräften an

Beruflichen Oberschulen - eine fächervergleichende Analyse....

Christian Schmidt

Die Öffnung des Hochschulzugangs für beruflich Gebildete: Förderung studienrelevanter Schlüsselkompetenzen in der Studieneingangsphase am Beispiel der Universität Kassel

\section{Teil IV: Schulentwicklungsforschung}

Marc Casper, Bernadette Dilger, Frederik Fischer, Katharina Fütterer, Nicole Naeve-Stoß und Tade Tramm

Entwicklung beruflicher Schulen im regionalen Verbund.....

Herausgeberschaft.

Autorinnen und Autoren 


\title{
Orientierungspotenziale des Basiscurriculums der Berufs- und Wirtschaftspädagogik für die disziplinäre Binnen- und Außenlegitimität
}

\author{
Matthias Söll
}

\section{Einleitung}

Der 1999 initiierte Bologna-Prozess zielt auf die Entwicklung transparenter und vergleichbarer Studiengänge $a b$, die in einem einheitlichen europäischen Hochschulraum eine hohe Studierendenmobilität begünstigen sollen (vgl. o.V. 1999, 1 ff.). Um die Qualität der tertiären Bildungsgänge hinsichtlich dieser Maßstäbe zu sichern und zu entwickeln, werden zahlreiche formelle Vorgaben erlassen. Zur Gewährleistung deren Umsetzung erfolgt zudem die Etablierung eines Akkreditierungssystems (vgl. KMK 2002, 4 ff.).

Die erziehungswissenschaftliche Teildisziplin Berufs- und Wirtschaftspädagogik konkretisiert die Richtlinien der Hochschulreformen, indem sie Standards für das von ihr verantwortete Studienfach erarbeitet. Im Basiscurriculum der Sektion Berufs- und Wirtschaftspädagogik der Deutschen Gesellschaft für Erziehungswissenschaft (Sektion BWP der DGfE) gibt die Disziplin an, dass sie sich bei der Studiengangentwicklung an den Grundsätzen des Bologna-Prozesses orientiert: „Mit dem Basiscurriculum verbindet sich [...] die Erwartung, dass lokale Lehrprogramme auf einen einheitlichen Rahmen bezogen werden und in ihren wesentlichen Inhalten übereinstimmen" (Sektion BWP der DGfE 2014, 6). Das Statut wurde im Jahr 2003 veröffentlicht und im Jahr 2014 aktualisiert (vgl. Sektion BWP der DGfE 2003, 2014).

Vor diesem Hintergrund steht die Entwicklung von Studiengängen regelmäßig im Fokus hochschulpolitischer Diskurse. Diese werden allerdings selten auf Basis empirischer Forschungsarbeiten geführt, sodass sie meist normativ oder subjektiv geprägt sind (vgl. Zlatkin-Troitschanskaia \& Kuhn 2010, 1). Pätzold (2012, 12 ff.) konstatiert, dass dies auch für die Studiengänge der Berufs- und Wirtschaftspädagogik gilt.

Um eine Grundlage zur empiriegestützen Entwicklung wirtschaftspädagogischer Studiengänge zu schaffen, wurde im Rahmen einer Curriculumanalyse eine entsprechende Vollerhebung durchgeführt. Die Untersuchung der Kernelemente der 113 ermittelten, profilierten Bachelor- und Masterstudiengänge an 30 Universitätsstandorten zeigt, dass sich deren Ziele zwar ähneln, ihre Strukturen und Inhalte allerdings teils erheblich voneinander sowie von den Vorgaben der Studiengangentwicklung abweichen (vgl. Söll 
2016, 266 ff.). Für konsekutive Studiengänge ist im Basiscurriculum bspw. ein Umfang von 75 Leistungspunkten (LP) für die Inhalte der Berufs- und Wirtschaftspädagogik vorgesehen (vgl. Sektion BWP der DGfE 2014, 12). Diese Norm erreichen derzeit 65,2 \% der untersuchten Bildungsgänge (vgl. Söll 2016, 264). Im Kontext der skizzierten Rahmenbedingungen der Entwicklung von Studiengängen sollte die stark eingeschränkte Transparenz und Vergleichbarkeit überraschen, zumal $76,3 \%$ der wirtschaftspädagogischen Studiengänge bereits erfolgreich akkreditiert wurden (vgl. Söll 2016, 94).

Während die Institutionen und Organisationen, die formelle Vorgaben zur Studiengangentwicklung erlassen, insbesondere die Erhöhung der Qualität und Kompatibilität von Bildungsprozessen und -abschlüssen anvisieren, fokussieren wissenschaftliche Disziplinen darüber hinaus auch auf deren Profilierung (vgl. Brand \& Tramm 2002, 266 f.). So setzt sich die Berufsund Wirtschaftspädagogik im Zuge der Formulierung von Studienstandards auch mit ihrem disziplinären Selbstverständnis auseinander (vgl. Sektion BWP der DGfE 2003, 1f. sowie 2014, 1 f.). Aus wissenschaftssoziologischer Perspektive dienen diese Debatten und ihre Ergebnisse der Konstituierung einer starken disziplinären Binnen- und Außenlegitimität (vgl. Lepsius 1973, 105 ff.).

Im Hinblick auf die Entwicklung des heterogenen Systems wirtschaftspädagogischer Studiengänge ist im vorliegenden Aufsatz die Frage handlungsleitend, welche Orientierungspotenziale die Genese des Basiscurriculums für das Innen- und Außenverhältnis der Disziplin Berufs- und Wirtschaftspädagogik bietet. Den Ausgangspunkt der Darstellungen bilden die vielschichtigen Rahmenbedingungen der Studiengangentwicklung, die in Kapitel 2 systematisiert werden. Im Zentrum des Beitrags stehen in Kapitel 3 dann die Entwicklung, die Ziele, die Orientierungsrahmen und der Aufbau der Basiscurricula sowie deren Regelungen zu den Zielen, Strukturen und Inhalten berufs- und wirtschaftspädagogischer Studiengänge. In Kapitel 4 schließlich werden die Orientierungspotenziale der Implementation der Basiscurricula der Berufs- und Wirtschaftspädagogik für die disziplinäre Binnen- und Außenlegitimität diskutiert.

\section{Formelle Rahmenbedingungen der Studiengangentwicklung}

Diplom- und Staatsexamensstudiengänge basierten auf staatlich genehmigten Rahmenordnungen und Vorgaben. Für die Entwicklung von Bachelor- und Masterstudiengängen ist gemäß der Grundsätze des New Public Managements das teil- bzw. nichtstaatliche Akkreditierungssystem verantwortlich (vgl. KMK 2002, 5; Wolter \& Kerst 2008, 137 ff.). Bisher wurde für die 
Entwicklung von Studiengängen allerdings kein einheitliches Verfahren eingeführt. Den Ausgangspunkt entsprechender Leitfäden, bspw. „Zehn Schritte nach ,Bologna“" (vgl. HRK 2004, $10 \mathrm{ff}$ ), bildet die Sichtung von formellen Rahmenbedingungen der Studiengangentwicklung.

Zur Entwicklung berufs- und wirtschaftspädagogischer Studiengänge liegt eine aktuelle, umfassende Übersicht mit Analyse der relevanten Vorgaben vor (vgl. Söll 2016, 79 ff. und 133 ff.). Diese geht von den Landeshochschulgesetzen als rechtlichen Basen der Studiengangentwicklung aus. Demnach werden fachspezifische Studiengänge wie die der Betriebswirtschaftslehre oder der Mathematik von (1.) den Landeshochschulgesetzen, (2.) den ländergemeinsamen Strukturvorgaben für die Akkreditierung, (3.) deren Auslegung durch den Akkreditierungsrat, (4.) den landesspezifischen Strukturvorgaben für die Akkreditierung, (5.) den Regeln für die Akkreditierung, (6.) den Vorgaben der Akkreditierungsagenturen und (7.) dem Qualifikationsrahmen für Deutsche Hochschulabschlüsse geregelt. Zudem gelten für lehramtsspezifische Studiengänge (1.) rechtliche Regelungen der Bundesländer, ${ }^{1}$ (2.) die Rahmenvereinbarung über die Ausbildung und Prüfung für ein Lehramt der Sekundarstufe II (berufliche Fächer) oder für die beruflichen Schulen (Lehramtstyp 5), (3.) die Standards für die Lehrerbildung in den Bildungswissenschaften und (4.) die ländergemeinsamen inhaltlichen Anforderungen für die Fachwissenschaften und Fachdidaktiken. Bei der Konzeption berufs- und wirtschaftspädagogischer Bildungsgänge fordert die Sektion BWP der DGfE $(2014,3)$ darüber hinaus die Berücksichtigung des rechtlich nicht bindenden Basiscurriculums.

Das Ergebnis der Recherche zeigt zunächst, dass bei der Studiengangentwicklung eine Vielzahl an Vorgaben zu berücksichtigen ist. Diese werden von den gesetzgebenden Instanzen des Bundes und der Länder sowie der Kultusministerkonferenz (KMK), der Hochschulrektorenkonferenz (HRK), dem Wissenschaftsrat und dem Akkreditierungsrat erlassen. Die Analyse der Dokumente in Bezug auf die Regelungen der Ziele, Strukturen, Inhalte und Rahmenbedingungen der Studiengänge zeichnet ein äußerst heterogenes Bild. Zwar können in den Vorgaben, die unterschiedliche Schwerpunkte setzen, grundsätzliche Gemeinsamkeiten in Bezug auf die Festlegung der Konturen des Studiums identifiziert werden, jedoch finden sich auch zahlreiche widersprüchliche Regelungen, bspw. zur strukturellen und inhaltlichen Gestaltung. Auch die Konzepte, auf denen die Vorgaben basieren, bspw. das Kompetenzkonzept, unterscheiden sich zum Teil voneinander.

1 Von den 14 Bundesländern, in denen bspw. das Studium der Wirtschaftspädagogik angeboten wird, haben sieben Lehrerbildungsgesetze und zwei spezifische Verordnungen erlassen. In fünf Bundesländern werden die Studiengänge ohne landesrechtliche Vorgaben konzipiert. Nach Walm und Wittek $(2014,16)$ zeigt sich daran die unterschiedliche Bedeutung, welche die Bundesländer der Lehrerbildung zuschreiben. 
Die Vorgaben zu fachspezifischen Studiengängen beinhalten keine fachlichinhaltlichen Standards, da diese bei der Akkreditierung im Rahmen von PeerReview-Verfahren durch geeignete Gutachter zu prüfen sind. Dies steht im Einklang mit dem durch das New Public Management forcierten Paradigmenwechsel von einer Input- zu einer Outcome-Steuerung (vgl. KMK 2002, 5; Wildt 2007, 44 f.). Dagegen finden sich in den Richtlinien zu lehramtsspezifischen Studiengängen zahlreiche Regelungen zu Studieninhalten. Überdies entsenden die für diese Bildungsgänge verantwortlichen Ministerien häufig Staatsvertreter mit Vetorecht in die Gutachtergruppen der Akkreditierungsverfahren. Somit wird das Ziel der staatlichen Deregulierung der Entwicklung von Studiengängen des Lehramts konterkariert (vgl. Reuter 2007, 124). Insgesamt ist davon auszugehen, dass sich dieses unsystematische Geflecht an Vorgaben eher hemmend als fördernd auf die Orientierung bei der Entwicklung transparenter und vergleichbarer Studiengänge auswirkt.

\section{Basiscurriculum für das universitäre Studienfach Berufs- und Wirtschaftspädagogik}

Die Basiscurricula für das universitäre Studienfach Berufs- und Wirtschaftspädagogik dienen einerseits der Steigerung der Qualität und Kompatibilität der entsprechenden Studienprozesse und -abschlüsse. Andererseits fokussiert die Berufs- und Wirtschaftspädagogik auch auf die Schärfung des disziplinären Profils. Im Folgenden werden die Genese, die Intentionen, die Leitgedanken und das Grundgerüst der Basiscurricula sowie deren Regelungen zu den curricularen Kernelementen berufs- und wirtschaftspädagogischer Studiengänge untersucht. Dabei steht die Frage im Mittelpunkt, inwiefern die Entwicklung der Richtlinie zur Konstituierung einer starken disziplinären Binnen- und Außenlegitimität beitragen kann.

\subsection{Genese}

Neben den Studienstrukturen stand an der Wende zum 21. Jahrhundert auch die inhaltliche Ausrichtung von Studiengängen zur Diskussion. Die Implementation eines Kerncurriculums wurde bspw. vom Wissenschaftsrat (2001, 29) als eine zentrale Reformaufgabe in der Lehrerbildung ausgewiesen. Kernoder Basiscurricula können als Ergebnisse der Kanonisierung von Bildung betrachtet werden (vgl. Tenorth 2004, 651 ff.). Zahlreiche Erziehungswissenschaftlerinnen und Erziehungswissenschaftler debattierten in dieser Zeit intensiv über ein solches Instrument (vgl. bspw. die Beiträge in der ,Zeitschrift für Pädagogik“ 45 [1999] 5 und in der Zeitschrift „Erziehungswissen- 
schaft" 15 [2004] 28). Auch die Vertreterinnen und Vertreter der Berufs- und Wirtschaftspädagogik beschäftigten sich mit dieser Thematik, wenngleich sich entsprechende Positionierungen seltener in der Literatur niederschlugen (vgl. Buchmann \& Kell 2001, 96 f.; Beck 2002, 124 f.).

Beck $(2002,125$ f.) legt die Funktion eines Kerncurriculums für Lehrerinnen und Lehrer an berufsbildenden Schulen aus professionssoziologischer Perspektive offen. Die Lehrenden erbringen als Professionsangehörige eine spezifische gesellschaftliche Leistung. Dabei greifen sie auf einen gemeinsamen Wissensbestand zurück. Dessen „Kern“ muss in der Lehrerbildung durch ein entsprechendes „Curriculum“ - ein Kerncurriculum - entwickelt und gesichert werden.

Das unter der Federführung von Beck und Zabeck erarbeitete Basiscurriculum für das universitäre Studienfach Berufs- und Wirtschaftspädagogik im Folgenden auch 1. Basiscurriculum - wurde im Jahr 2003 verabschiedet (vgl. Sektion BWP der DGfE 2003, 1; Sloane 2003, 490). ${ }^{2}$ Gut zehn Jahre später erfolgte die Bewilligung des durch Weber, Kremer, Tenberg, Weyland und Beck überarbeiteten Grundsatzpapiers - im Folgenden auch 2. Basiscurriculum (vgl. Sektion BWP der DGfE 2014, 2 ff.).

Die Curricula beruhen auf intradisziplinären, öffentlichen Diskursen zwischen den Fachvertreterinnen und Fachvertretern. Darüber hinaus wurden die berufs- und wirtschaftspädagogische Lehre und Forschung, die rechtlichen Rahmenbedingungen der Studiengänge sowie die Anforderungen von Tätigkeiten, die Absolventinnen und Absolventen nach dem Studium in der Regel ausführen, analysiert (Sektion BWP der DGfE 2003, 1 ff. sowie 2014, 1 ff.).

\subsection{Ziele}

Grundsätzlich markieren die Curricula Eckpunkte des disziplinären Selbstverständnisses und setzen inhaltliche Standards (Sektion BWP der DGfE 2003, 1 f. sowie 2014, 1 f.). Durch die Verständigung auf eine gemeinsame Identität zielen die Richtlinien bezüglich des disziplinären Innenverhältnisses einerseits auf die Professionalisierung der Berufs- und Wirtschaftspädagogik selbst ab. Andererseits sichern und entwickeln sie hinsichtlich des disziplinären Außenverhältnisses durch die Vorgabe inhaltlicher Standards die Qualität des universitären Studiums der Berufs- und Wirtschaftspädagogik, das die Professionalität der Absolventinnen und Absolventen entwickeln soll.

2 Das Basiscurriculum wurde anfangs auch als Kerncurriculum bezeichnet (vgl. Bank 2009, 4; Sloane 2003, 490). Die DGfE veröffentlichte im Jahr 2008 allerdings selbst ein Kerncurriculum für erziehungswissenschaftliche Haupt- und Nebenfachstudiengänge sowie für Lehramtsstudiengänge (vgl. DGfE 2010, 7). Die Differenzierung der Bezeichnungen soll einer Verwechslung vorbeugen. 


\subsection{Orientierungsrahmen}

Beck (2005, 5 und 10) plädiert ausdrücklich für ein professionalitätsförderndes, input-orientiertes Basiscurriculum. In einem Brückenschlag zwischen Input- und Outcome-Orientierung sieht der Autor die Gefahr der Marginalisierung von Inhalten. Aufgrund der häufig fehlenden Forschungsergebnisse über die Zusammenhänge zwischen Input und Outcome könne eine Systematisierung, bspw. in einer Matrix, nur spekulativ erfolgen. Daraus resultiere die Gefahr der systematischen Unter- oder Überschätzung des Geltungsanspruchs der Inhalte. Dies führe bezüglich der Entwicklung berufs- und wirtschaftspädagogischer Professionalität zu Qualitätseinbußen (vgl. Beck 2005, 14 f.).

Sloane (2003, 490 f.) und Wilbers (2004, 11 f.) machten dagegen bereits zur Zeit der Einführung des Basiscurriculums auf die Problematik aufmerksam, dass lehrerbildende Studiengänge vor dem Hintergrund der vorherrschenden Bildungs- und Hochschulpolitik standard- und kompetenzbasiert gestaltet werden sollten. Als Lösung schlägt Sloane (2003, 490 f.) die von Beck (2005, 5 und 10) abgelehnte, systematische Zusammenführung der fachwissenschaftlichen Struktur der Berufs- und Wirtschaftspädagogik mit den sich an Outcomes orientierenden Standards der Lehrerbildung vor. Auch Brand und Tramm (2002, 269 ff.) setzen sich für solch eine integrative Lösung ein.

Zur Förderung berufs- und wirtschaftspädagogischer Professionalität rekurriert die erste Fassung des Basiscurriculums auf inhaltliche Standards (vgl. Sektion BWP der DGfE 2003, 1), während die zweite diesbezüglich einen kompetenzorientierten Raum (vgl. Sektion BWP der DGfE 2014, 2) aufspannt. Allerdings wird in dem letztgenannten Statut nicht allein auf die Förderung von Kompetenz fokussiert. Indem auch die zentralen Inhalte der Berufs- und Wirtschaftspädagogik explizit genannt und strukturell verortet werden, haben sie weiterhin ein starkes Gewicht. Somit ist das ursprüngliche Curriculum eher wissenschaftsorientiert, das überarbeitete eher situationsund persönlichkeitsorientiert.

\subsection{Aufbau}

Die beiden Richtlinien ähneln sich hinsichtlich ihres Aufbaus, der in Tabelle 1 veranschaulicht wird. In den Kapiteln 1 und 2 wird in die Basiscurricula eingeführt. Darauf folgt die Formulierung von Leitbildern (Kapitel 3) und Professionalitätsdimensionen (Kapitel 4). Die in den Kapiteln 5 systematisierten inhaltlichen Schwerpunkte der Studiengänge werden allerdings nur im 1. Basiscurriculum in Kapitel 6 spezifiziert. Beide Statute enden mit Implementationsgrundsätzen (1. Basiscurriculum: Kapitel 7; 2. Basiscurriculum: 
Kapitel 6). Die unterschiedlichen Orientierungen der Curricula spiegeln sich auch in den Umfängen der einzelnen Kapitel wider. Im eher wissenschaftsorientierten 1. Basiscurriculum nehmen die Kapitel 5 und 6, die Studieninhalte präzisieren, einen Umfang von 58,6\% ein. ${ }^{3}$ Der mit diesen Kapiteln im 2. Basiscurriculum korrespondierende Teil 5 hat dagegen nur einen Umfang von $12,6 \%$. Themen wie künftige berufliche Tätigkeitsfelder, professionelles Handeln, dafür notwendige Dispositionen und spezifische Kompetenzprofile werden im 1. Basiscurriculum in den Kapiteln 3 und 4 mit einem Umfang von 9,4\% abgedeckt. Diese Gegenstände nehmen im 2. Basiscurriculum in den Kapiteln 3, 4 und 5 dagegen 54,3\% des Gesamtumfangs ein.

Tab. 1: Aufbau der Basiscurricula

\begin{tabular}{|c|c|c|c|c|c|c|c|}
\hline Nr. & $\begin{array}{l}\text { Basiscurriculum } \\
2003\end{array}$ & $\sum \mathbf{Z} .:$ & $\%$ & Nr. & $\begin{array}{l}\text { Basiscurriculum } \\
2014\end{array}$ & $\sum \mathbf{Z} .:$ & $\%$ \\
\hline 1 & Vorwort & 6.391 & 17,5 & 1 & Vorwort & 8.966 & 26,7 \\
\hline 2 & Präambel & 3.957 & 10,8 & 2 & Geltungsbereich & 5.085 & 15,1 \\
\hline 3 & Leitbild & 2.145 & 5,9 & 3 & Leitbild & 4.172 & 12,4 \\
\hline 4 & $\begin{array}{l}\text { Professionalitäts- } \\
\text { dimensionen }\end{array}$ & 1.265 & 3,5 & 4 & $\begin{array}{l}\text { Professionalitäts- } \\
\text { dimensionen }\end{array}$ & 9.840 & 29,3 \\
\hline 5 & Rahmenstruktur & 1.884 & 5,2 & 5 & $\begin{array}{l}\text { Hinweise für } \\
\text { Kompetenzprofile }\end{array}$ & 4.232 & 12,6 \\
\hline 6 & Lehrinhalte & 19.505 & 53,4 & 6 & $\begin{array}{l}\text { Implementations- } \\
\text { grundsätze }\end{array}$ & 1.346 & 4,0 \\
\hline \multirow[t]{2}{*}{7} & $\begin{array}{l}\text { Implementations- } \\
\text { grundsätze }\end{array}$ & 1.393 & 3,8 & & & & \\
\hline & & 36.540 & & & & 33.641 & \\
\hline
\end{tabular}

Z.: Zeichen inklusive Leerzeichen (Erhebung mit Word 2013)

Quelle: Eigene Darstellung

3 In Kapitel 6 werden jedem Themengebiet auf 14 Seiten in tabellarischer, stichpunktartiger Form durchschnittlich 7,9 Unterpunkte (Min=3; $M a x=12 ; S D=2,6$ ), die zum Teil nochmals untergliedert sind, zugeordnet. Das ursprünglich veröffentlichte Kerncurriculum ähnelt mit Ausnahme des Vorworts dem 1. Basiscurriculum. Es wurde allerdings um einen Anhang ergänzt, der zu den Kapiteln 5 und 6 ca. 750 Quellen ausweist. 


\subsection{Studienziele}

Gemäß der Leitbilder der Basiscurricula sollen Absolventinnen und Absolventen des berufs- und wirtschaftspädagogischen Studiums in der Lage sein,

„-- Erkenntnisse im Bereich der Berufs- und Wirtschaftspädagogik theoriegeleitet und systematisch zu erarbeiten,

- praktische Fragen und Probleme in den genannten Tätigkeitsfeldern theoriegeleitet und kritisch zu reflektieren sowie

- theoriegeleitet begründete, auf individuelle und kollektive Bedürfnisse abgestimmte Lösungen zu entwickeln und umzusetzen“ (Sektion BWP der DGfE 2014, 7; vgl. Sektion BWP der DGfE 2003, 6).

Diese professionelle, ethisch-reflexive Handlungskompetenz ist im Sinne lebenslangen Lernens stetig weiterzuentwickeln (vgl. Sektion BWP der DGfE 2014, 7 f. sowie 2003, 6). Es werden zudem typische Tätigkeitsfelder wie das berufsbildende Schulwesen, das betriebliche Bildungs- und Personalwesen oder die berufs- und wirtschaftspädagogische Forschung und Lehre sowie mit diesen korrespondierende professionelle Handlungen wie die Curriculumentwicklung, die Gestaltung von Lehr-Lern-Prozessen oder die Entwicklung der Rahmenbedingungen der beruflichen Bildung systematisiert (Sektion BWP der DGfE 2003, 6 sowie 2014, 6 ff.).

\subsection{Studienstruktur}

Das im Jahr 2003 eingeführte Basiscurriculum bezieht sich auf grundständige Studiengänge mit Diplom- oder Staatsexamensabschlüssen (vgl. Sektion BWP der DGfE 2003, 3 und 26). Für diese wurden insgesamt 160 Semesterwochenstunden (SWS) veranschlagt (vgl. KMK 1999, 22). Dabei soll das Fach Berufs- und Wirtschaftspädagogik inklusive der fachspezifischen Didaktik der jeweiligen beruflichen Fachrichtung ca. 40 SWS umfassen (vgl. Sektion BWP der DGfE 2003, 5). Dies entspricht einem Studienanteil von 25 Prozent.

Die im Rahmen des Bologna-Prozesses eingeführte Studienstruktur bildet den Ausgangspunkt des überarbeiteten Basiscurriculums aus dem Jahr 2014 (vgl. Sektion BWP der DGfE 2014, 3). Bei einem Bachelor- und Masterstudium sind insgesamt 300 LP zu erwerben (vgl. KMK 2010, 3). In dem Grundsatzdokument der Sektion BWP wird für das Fach Berufs- und Wirtschaftspädagogik ein Umfang von ca. 75 LP gefordert (vgl. Sektion BWP der DGfE 2014, 12). Der anvisierte fachspezifische Studienanteil beträgt somit ebenfalls 25 Prozent. 


\subsection{Studieninhalte}

Die Basiscurricula weisen jeweils fünf Studienschwerpunkte mit dazugehörigen Themengebieten aus:

- Didaktische Inhalte (1. Basiscurriculum: „Didaktik der beruflichen Ausund Weiterbildung“" [26,7\%]; 2. Basiscurriculum: „Berufliches Lehren, Lernen, Entwickeln“ [25,0 \%]) nehmen in beiden Curricula ca. 25,0 \% des Fachs ein.

- Auf die Auseinandersetzung mit der Berufspraxis (1. Basiscurriculum: „Unterrichts- und unterweisungspraktische Studien“ [13,3\%]; 2. Basiscurriculum „Berufs- und wirtschaftspädagogische Praxisfelder“ [15,0 $\%$ ]) entfallen jeweils ungefähr 15,0 \% dieses Studienteils.

- Die Schwerpunkte „Grundlagen der Berufs- und Wirtschaftspädagogik“ $(20,0 \%)$ und "Bedingungen und Strukturen beruflichen Lernens“ $(26,7 \%)$ wurden im Zuge der Überarbeitung des Curriculums in ,Theorien, Organisation, Strukturen beruflicher Bildung“ $(25,0 \%)$ und „Professionalisierung" $(10,0 \%)$ umstrukturiert. Dies ging mit einer Reduzierung der Studienanteile von insgesamt $46,7 \%$ auf $35,0 \%$ einher.

- Der Bereich „Forschungsmethoden“ wurde somit von knapp 15,0 \% auf 25,0 \% (1. Basiscurriculum: „Ansätze und Methoden der quantitativen und qualitativen Berufsbildungsforschung “ $[13,3 \%] ; 2$. Basiscurriculum: „Forschungsmethoden“ [25,0\%]) deutlich aufgewertet (vgl. Sektion BWP der DGfE 2003, 8 sowie 2014, 12).

\section{Zusammenfassung und Fazit}

Die Basiscurricula der Berufs- und Wirtschaftspädagogik spiegeln in Bezug auf das korrespondierende Studium zum einen die vorherrschenden Normen, Werte und Einstellungen berufs- und wirtschaftspädagogischer Fachvertreterinnen und Fachvertreter wider. Zum anderen regeln sie die Ziele, Strukturen, Inhalte und Rahmenbedingungen der entsprechenden Studiengänge. Während das erste, eher wissenschaftsorientierte Statut auf die inhaltliche Dimension des Studiums fokussiert, konzentriert sich das zweite, eher situations- und persönlichkeitsorientierte, auf dessen Zieldimension. Abschließend werden die Orientierungspotenziale der Curricula vor dem Hintergrund der Konstituierung der disziplinären Binnen- und Außenlegitimität der Berufs- und Wirtschaftspädagogik diskutiert.

Die Vielzahl an formellen Vorgaben ist für die Entwicklung transparenter und vergleichbarer Studiengänge eher ab- als zuträglich. Bei der Analyse der Inhalte der in Kapitel 2 systematisierten Reglementierungen fällt aller- 
dings auf, dass sich diese überwiegend auf unterrichts- und schulnahe Tätigkeiten beziehen. Das zentrale Prinzip der Polyvalenz berufs- und wirtschaftspädagogischer Bachelor- und Masterstudiengänge ist lediglich in den ländergemeinsamen inhaltlichen Anforderungen für die Fachwissenschaften und Fachdidaktiken verankert. Dagegen war dieser Grundsatz zentraler Bestandteil der Diplomstudiengänge regelnden Rahmenordnung (vgl. Söll 2016, 133 ff.). In Bezug auf die Regelungsebene kann konstatiert werden, dass die Bedeutung des polyvalenten Studiengangmodells zugunsten des lehrerbildenden abgenommen hat (vgl. Söll 2016, 163 f.). Hinsichtlich des disziplinären Außenverhältnisses ist es folglich durchaus sinnvoll, die eigenen Prinzipien in einem konsistenten Basiscurriculum nach außen zu kommunizieren und dessen Berücksichtigung bei der Studiengangentwicklung sowie bei der Akkreditierung einzufordern.

Sloane $(2003,490$ f.) und Wilbers $(2004,15)$ weisen darauf hin, dass die Richtlinie regelmäßig an bildungs- und hochschulpolitische Rahmenbedingungen, wie die gestufte Studienstruktur mit Bachelor- und Masterabschlüssen oder die Outcome-Orientierung, angepasst werden muss. Fehlen entsprechende Bezüge, droht eine partielle Umsetzung des Statuts und damit die Verfehlung der ursprünglichen Ziele. Ferner besteht die Gefahr, dass sich Studiengangentwicklerinnen und -entwickler an generellen Vorgaben orientieren müssen, wenn sie ihre Spezifika nicht in den aktuellen Diskurs einbringen. Das Ergebnis einer explorativ angelegten Interviewstudie zu den Orientierungen von 14 Professorinnen und Professoren der Wirtschaftspädagogik kann als Indiz für diese Befürchtungen interpretiert werden. Es zeigt sich, dass die Befragten das sich auf Diplom- und Staatsexamensstudiengänge beziehende 1. Basiscurriculum bei der Ableitung von Zielen (21,4\%), Strukturen, Rahmenbedingungen $(7,1 \%)$ und Inhalten $(50,0 \%)$ von Bachelor- und Masterstudiengängen nur vereinzelt als Richtlinie nutzten (vgl. Söll 2016, 208 ff.). Hinsichtlich der disziplinären Außenlegitimität rechtfertigen diese Argumente die Implementation der gestuften Studienstruktur sowie der Outcome-Orientierung im überarbeiteten Basiscurriculum.

Bei der Modifikation der Vorgaben muss allerdings berücksichtigt werden, dass deren Orientierungspotenzial bei der Gestaltung von Studiengängen sinken kann, wenn die Beschreibungen der Kernbestandteile der Bildungsgänge einen zu hohen Abstraktionsgrad aufweisen. Diesbezüglich befürchtet Beck (2005, 14 f.), die Entwicklung berufs- und wirtschaftspädagogischer Professionalität werde ,im Kompetenzkorsett verkümmern“ (Beck 2005, 15). Einerseits spricht die Berücksichtigung aktueller bildungs- und hochschulpolitischer Rahmenbedingungen im 2. Basiscurriculum dafür, dass dieses bei der Ableitung von Studienzielen, -strukturen und -rahmenbedingungen mehr Orientierung bietet. Andererseits wirkt sich die starke Reduzierung der Präzisierung von Studieninhalten zugunsten der Beschreibung eines kompetenz- 
orientierten Raums möglicherweise negativ auf das Orientierungsvermögen der Leitlinie aus.

Die Entwicklung von Basiscurricula entfaltet ihre Wirkung auch in Bezug auf das disziplinäre Innenverhältnis. Aushandlungsprozesse zwischen den Vertreterinnen und Vertretern der Berufs- und Wirtschaftspädagogik über die Ziele, Inhalte, Strukturen und Rahmenbedingungen des Studiums kennzeichnen eine lebendige wissenschaftliche Disziplin (vgl. Reinisch 2009, 9 und 13 f.). Dabei müssen die Vorgaben auch hinsichtlich empirischer Erkenntnisse zur Studiengangentwicklung diskutiert werden. So zeigt die Untersuchung der Inhaltsbereiche konsekutiver wirtschaftspädagogischer Studiengänge, dass Forschungsmethoden bspw. durchschnittlich lediglich einen Umfang von 8,8 \% einnehmen (vgl. Söll 2016, 261). Deren Bedeutung ist im aktuellen Basiscurriculum mit einem Studienanteil von 25 Prozent dagegen deutlich größer. ${ }^{4}$

Die sich an entsprechende Diskurse anschließende Einigung auf ein Basiscurriculum als Konsens über den normativen, strukturellen und inhaltlichen Rahmen für berufs- und wirtschaftspädagogische Studiengänge lässt sich als Stärkung der disziplinären Binnenlegitimität interpretieren. Diese könnte noch intensiviert werden, indem die Auseinandersetzungen in einschlägigen Publikationsorganen veröffentlicht würden. Da an der Binnenlegitimität der Disziplin ebenfalls kontinuierlich weitergearbeitet werden muss, ist die Aktualisierung des Basiscurriculums auch aus innerdisziplinärer Perspektive angezeigt.

Die Analyse der Entwicklungs- und Implementationsprozesse der Basiscurricula zeigt, dass sie sowohl für die Binnen- als auch für die Außenlegitimität der Berufs- und Wirtschaftspädagogik große Orientierungspotenziale bieten. Vor diesem Hintergrund sind weitere Forschungsarbeiten von Interesse, welche die Orientierungsleistungen des Basiscurriculums für die Binnenund Außenlegitimität der Disziplin in den Blick nehmen.

\section{Literatur}

Bank, V. (2009): Berufs- und Wirtschaftspädagogik: Epitaph einer Disziplinlosen. In: bwp@Berufs- und Wirtschaftspädagogik - online 16, 1-22. Online: http://www. bwpat.de/content/uploads/media/bank_bwpat16.pdf (28.11.2016).

4 Die starke Betonung der Forschungsmethoden kann als Versuch interpretiert werden, den Schaden für berufs- und wirtschaftspädagogische Studiengänge abzumildern, der durch die starke staatliche Regulierung entsteht. Diese führt nach Sloane (2010, 4 f.) des Öfteren dazu, dass die Studiengänge von der Scientific Community nicht als forschungsorientiert und somit nicht als gleichwertig zu anderen Studiengängen angesehen werden. 
Beck, K. (2002): Plädoyer für ein Kern-Curriculum zur Ausbildung von Lehrerinnen und Lehrern an berufsbildenden Schulen. Zeitschrift für Berufs- und Wirtschaftspädagogik, 98 (1), 124-130.

Beck, K. (2005): Standards für die Ausbildung von Berufsschullehrern in Europa Professionalisierung im Kompetenzkorsett? Johannes Gutenberg-Universität Mainz. Mainz (Arbeitspapiere WP, 52). Online: http://www.wipaed.uni-mainz.de /ls/ArbeitspapiereWP/gr_Nr.52.pdf (28.11.2016).

Brand, W.; Tramm, T. (2002): Notwendigkeit und Problematik eines Kerncurriculums für die Ausbildung von Berufs- und Wirtschaftspädagogen. In: S. Baabe, E. M. Haarmann \& I. Spiess (Hrsg.): Für das Leben stärken - Zukunft gestalten. Behindertenpädagogische, vorberufliche und berufliche Bildung - Verbindungen schaffen zwischen Gestern, Heute und Morgen, (S. 266-277). Paderborn: Eusl.

Buchmann, U.; Kell, A. (2001): Abschlußbericht zum Projekt Konzepte zur Berufsschullehrerbildung im Auftrag des Bundesministeriums für Bildung und Forschung (Förder-Kennzeichen: K 61.16.00). Siegen.

DGfE (2010): Kerncurriculum Erziehungswissenschaft. Empfehlungen der Deutschen Gesellschaft für Erziehungswissenschaft (DGfE). Opladen, Farmington Hills: Verlag Barbara Budrich (Mitteilungen der DGfE, 21).

HRK (2004): Bologna-Reader I. Texte und Hilfestellungen zur Umsetzung der Ziele des Bologna-Prozesses an deutschen Hochschulen. Bonn: HRK (Beiträge zur Hochschulpolitik, 8). Online: http:/www.hrk.de/fileadmin/redaktion/hrk/02Dokumente/02-10-Publikationsdatenbank/Beitr-2004-08_Bologna-Reader_I.pdf (28.11.2016).

KMK (1999): Rahmenordnung für die Diplomprüfung im Studiengang Wirtschaftspädagogik an Universitäten und gleichgestellten Hochschulen. Online: http://www. kmk.org/fileadmin/veroeffentlichungen_beschluesse/1999/1999_03_ 19-RO-W-Paedagogik-HS.pdf (28.11.2016).

KMK (2002): Künftige Entwicklung der länder- und hochschulübergreifenden Qualitätssicherung in Deutschland. Online: http://www.kmk.org/fileadmin/veroeffentlichungen_beschluesse/2002/2002_03_01-Qualitaetssicherung-laender-hochschuluebergreifend.pdf (28.11.2016).

KMK (2010): Ländergemeinsame Strukturvorgaben für die Akkreditierung von Bachelor- und Masterstudiengängen. Online: http://www.kmk.org/fileadmin/veroeffentlichungen_beschluesse/2003/2003_10_10-Laendergemeinsame-Strukturvorgaben.pdf (28.11.2016).

Lepsius, R. M. (1973): Gesellschaftsanalyse und Sinngebungszwang. In: G. Albrecht, H. Daheim \& F. Sack (Hrsg.): Soziologie: Sprache, Bezug zur Praxis, Verhältnis zu anderen Wissenschaften. René König zum 65. Geburtstag, (S. 105-116). Opladen: Westdeutscher Verlag.

o. V. (1999): Der europäische Hochschulraum. Gemeinsame Erklärung der europäischen Bildungsminister. 19. Juni 1999. Bologna. Online: https://www.hrk.de/file admin/redaktion/hrk/02-Dokumente/02-03-Studium/02-03-01-Studium-Studienreform/Bologna_Dokumente/Bologna_1999.pdf (28.11.2016).

Pätzold, G. (2012): Lehrerbildung für berufsbildende Schulen als herausfordernde Gestaltungsaufgabe. In: M. Becker, G. Spöttl \& T. Vollmer (Hrsg.): Lehrerbildung in Gewerblich-Technischen Fachrichtungen, (S. 11-33). Bielefeld: Bertelsmann (Berufsbildung, Arbeit und Innovation, 37). 
Reinisch, H. (2009): Über Nutzen und Schaden des Philosophierens über das Selbstverständnis der Berufs- und Wirtschaftspädagogik. Anmerkungen aus wissenschaftssoziologisch inspirierter Sicht.bwp@Berufs- und Wirtschaftspädagogikonline 16, 1-18. Online: http:/www.bwpat.de/content/uploads/media/ reinisch_bwpat16.pdf (28.11.2016).

Reuter, L. R. (2007): Zur Akkreditierung von Studiengängen im Rahmen des Bologna-Prozesses am Beispiel erziehungswissenschaftlicher Studienprogramme. Erziehungswissenschaft, 18 (35), 116-125.

Sektion BWP der DGfE (2003): Basiscurriculum für das universitäre Studienfach Berufs- und Wirtschaftspädagogik. Jena. Online: http://www.bwp-dgfe.de/images /Dokumente/Basiscurriculum_BWP_040202.pdf (28.11.2016).

Sektion BWP der DGfE (2014): Basiscurriculum für das universitäre Studienfach Berufs- und Wirtschaftspädagogik im Rahmen berufs- und wirtschaftspädagogischer Studiengänge. Schwäbisch-Gmünd. Online: http://www.bwp-dgfe.de/ images/Dokumente/Basiscurriculum_Berufs-und_Wirtschaftspaedagogik_2014. pdf (28.11.2016).

Sloane, P. F. E. (2003): Bakkalaureaten und Magister für die (berufsbildenden) Schulen - Quo vadis Berufs- und Wirtschaftspädagogik. Zeitschrift für Berufs- und Wirtschaftspädagogik, 99 (4), 481-493.

Sloane, P. F. E. (2010): Avanti dilettanti: die Reform der Lehrerbildung. Zur Einführung des Masters of Education (M.Ed.) und der vielleicht vergeblichen Hoffnung auf Professionalität. Zeitschrift für Berufs- und Wirtschaftspädagogik, 106 (1), 110.

Söll, M. (2016): Die Entwicklung von Studiengängen. Eine Curriculumanalyse am Beispiel der Wirtschaftspädagogik. Detmold: Eusl (Wirtschaftspädagogisches Forum, 55).

Tenorth, H.-E. (2004): Bildungsstandards und Kerncurriculum. Systematischer Kontext, bildungstheoretische Probleme. Zeitschrift für Pädagogik 50 (5), 650-661.

Walm, M.; Wittek, D. (2014): Lehrer_innenbildung in Deutschland im Jahr 2014. Eine phasenübergreifende Dokumentation der Regelungen in den Bundesländern - Eine Expertise im Auftrag der Max-Traeger-Stiftung. GEW Gewerkschaft Erziehung und Wissenschaft. Online: https://www.gew.de/index.php?eID=dump File \&t=f\&f=23490\&token=270cc087a163e0ebb51f76950df2efa377f1d091\&sdown load $=\& n=$ Lehrer-Innenbildung_2014_A4_web.pdf (28.11.2016).

Wilbers, K. (2004): Standards für die Bildung von Lehrkräften. Arbeitsbericht. St. Gallen.

Wildt, J. (2007): Vom Lehren zum Lernen. In: F. Bretschneider \& J. Wildt (Hrsg.): Handbuch Akkreditierung von Studiengängen. Eine Einführung für Hochschule, Politik und Berufspraxis, (S. 44-54). Bielefeld: Bertelsmann (GEW-Materialien aus Hochschule und Forschung, 110).

Wissenschaftsrat (2001): Empfehlungen zur künftigen Struktur der Lehrerbildung. Berlin. Online: http://www.wissenschaftsrat.de/download/archiv/5065-01.pdf (28.11.2016).

Wolter, A.; Kerst, C. (2008): Akkreditierung als Verfahren der Qualitätssicherung von Studiengängen in Deutschland. Eine Policy-orientierte Analyse. In: E. Klieme \& R. Tippelt (Hrsg.): Qualitätssicherung im Bildungswesen, (S. 235-155). Weinheim [u. a.]: Beltz (Zeitschrift für Pädagogik, Beiheft 53). 
Zlatkin-Troitschanskaia, O.; Kuhn, C. (2010): Messung akademisch vermittelter Fertigkeiten und Kenntnisse von Studierenden bzw. Hochschulabsolventen. Analyse zum Forschungsstand. Johannes Gutenberg-Universität Mainz. Mainz (Arbeitspapiere WP, 56). Online: http:/www.wipaed.uni-mainz.de/ls/Arbeitspapiere WP/gr_Nr.56.pdf, (28.11.2016). 